\title{
Abstracts of the congress World Nutrition Rio2012
}

Organised by World Public Health Nutrition Association, Brazilian Association of Public Health and Rio de Janeiro State University

Rio de Janeiro State University, Brazil - April 27-30, 2012

\section{SPECIAL ISSUE EDITORS}

Inês Rugani Ribeiro de Castro

Barrie M. Margetts

Sabrina Ionata de Oliveira Granheim

\section{WORLD NUTRITION RIO2012 COMMITTEES}

\section{Executive Committee}

Barrie Margetts | President - WPHNA

Luis Augusto Facchini I President - ABRASCO

Catherine Geissler I WPHNA

Elisabetta Gioconda Iole Giovanna Recine I ABRASCO

Geoffrey Cannon । WPHNA

Fabio da Silva Gomes I ABRASCO

Harriet Kubnlein | WPHNA

Janine Giuberti Coutinho I ABRASCO

Sabrina Ionata de Oliveira Granbeim I WPHNA

Luciana Maria Cerqueira Castro I ABRASCO

Inês Rugani Ribeiro de Castro | Executive Secretary

\section{Executive Secretariat}

Inês Rugani Ribeiro de Castro I Executive Secretary Sabrina Ionata de Oliveira Granbeim I Deputy Secretary Anelise Rizzolo I Deputy Secretary

Thiago Barreto Bacellar Pereira | Assistant

\section{With advice and support from ABRASCO}

Carlos dos Santos Silva

Margareth Pessanha de Souza

\section{Programme Committee}

Catherine Geissler I Facilitator Fabio da Silva Gomes I Facilitator

Harriet Kubnlein I Facilitator

Janine Giuberti Coutinho I Facilitator

Andhressa Fagundes I Support

Anelise Rizzolo I Support

Bárbara de Alencar Teixeira | Support

Camila Maranha Paes de Carvalho I Support

Cláudia Roberta Bocca dos Santos I Support

Elisabetta G. Iole Giovanna Recine I Support

Gabriela Bioni e Silva I Support

Juliana Martins | Support

Kelly Poliany de Souza Alves I Support

Patricia Gentil I Support

\section{EDITORIAL ASSISTANTS}

Silvia Cristina Farias

Juliana Martins Oliveira

Paulo Cesar Pereira de Castro Junior

\section{Operational Committee}

Luciana Maria Cerqueira Castro | Facilitator Sabrina Ionata de Oliveira Granhein I Facilitator Silvia Cristina Farias I Assistant Amanda da Silva Franco I Support Ana Maria Mendes Monteiro Wandelli I Support Bruna Pitasi Arguelhes I Support Cláudia Roberta Bocca dos Santos I Support Daniel Alves de Oliveira I Support Elisa Proença da Silva Mendonça I Support Juliana Martins I Support Leticia Ferreira Tavares I Support Luiza Toschi I Support Maria Teixeira de Moraes I Support Marta Regina M. dos Santos Nehme I Support Patrícia Maria Périco Perez I Support Paulo Cesar Pereira de Castro Junior I Support Paulo Orlando Ribeiro da Silva I Support Raquel Mezzavilla I Support Rebecca Louise Greenwood I Support

\section{Congress advisors}

Agneta Yngve Claudio Schuftan

Kenneth Rochel Camargo Jr.

Mark Lawrence

Philip James

Rafael Moreira Claro

Reggie Annan

Roger Hughes

Sofia Sayuri Yoneta

Urban Jonsson 